\title{
Inculcating Practices of Social Interaction among Young Children for Better Mental Well Being
}

\author{
Sarmad Muhammad Soomar* \\ Aga Khan University School of Nursing \& Midwifery, Pakistan
}

*Corresponding author: Sarmad Muhammad Soomar, Aga Khan University School of Nursing \& Midwifery, Pakistan

\begin{abstract}
Young children have similar kind of stressors and that are impactful to their mental health. In this age it is very important to develop such practices that encourage social interaction. Inculcating social interaction practices and activities will empower the young generation to deal with their stressors and enhance their mental wellbeing. This paper will focus on sharing various strategies that will be helpful to all people dealing with young children at home or any kind of healthcare setup.
\end{abstract}

Keywords: Social Interaction; Mental Health; Wellbeing; Stressors; Young Children

\section{Introduction}

Mental health of young people is a distinct dealing, but it doesn't mean that it is difficult and impossible to deal. Youth requires motivation, social interaction, support, privacy and respect while they are dealt. In certain cases flaw in these characteristics lead them towards negative path and working individually on these characteristics with available and suggested resources it can help in a better way [1]. On the other hand stress is a phenomenon which is experience by everyone without respect of age [2]. The common stressors that are observed in terms of youth are universal like others but are more related to academics. Stress that is received from academic level can lead to substance abuse, insomnia, suicide and sometimes paranoia because of chemical imbalances [1,2]. All these problems ad stressors create a sense of disconnection from social interactions and youngsters end up into closing self into rooms, not talking to one or remain excluded at home [1]. As we see the overall it is common around the globe that social ties and norms have a great connection to one's life. It has also distinct relatability with our mental wellbeing especially the young children [1,3]. As young children have many personal and private issues that they really want to share with someone who can listen and understand them. No doubt the time which they are passing through keeps them away from family creating a personal space but it's essential to talk to them, as they require someone to listen $[3,4]$. Social interaction is necessary for young children to grew and learn life.
Their common social circles are in relatives, friends, people at the institution one studies with or works. When they increase their circles, when they get to know people; share things with them, time spent with them makes them happy [3]. But what happens when they think that issues are coming from those social circles. Young age is sensitive, and things can make them upset too quickly. In this the social interaction shouldn't be stopped as it will help them to grow and learn many aspects of living [4]. There are several more modes to their practices of social interaction. In the growing technologic growing area social interaction is limited to internet. Sitting at home and talking to friends is social interaction. The links with their social circles is weak, and that cannot improve their social practices and social interaction. This is commonly witnessed by many of us while working with children [5]. Furthermore, socially excluded individuals tend to remain at home, stay alone, function and live separate, have limited interactions and are less socialize with people around. These experiences may be developed because of illness, personal nature, bad experiences with people, lack of trust, issues in adaption with others; it depends on growth and development process as well [3,4]. Gender roles with young girls and boys also impact their social interaction. As in context of many third world countries, boys are expected to be more involve in interacting outside home with people because of education or earning. 
In contrast, young girls are expected to be at home for house chores or helping mothers. Also, their growing age and expectation of marriage by families restrict them to remain at home and interact less with social circles. Mainly the interaction is limited to family or friends of same gender [6]. Young children with mental health disorders epically schizophrenia lack social interaction because of their condition. Also witnessing from practices, we have observed that young children with anxiety disorders or phobia also avoid social interactions [4]. To improve their conditions and promote social interaction, social skills development trainings with effective practical activities are very helpful. This can include: social perception, conversational skills, information handling skills, assertiveness, and role and affiliation skills [7,1]. Few activities practiced in this social inclusion strategy include Facial emotion recognition, two way interaction practice, Identification of correct patterns of non-verbal communication, Two-way conversations with a manipulating friend trying to take advantage of the participant, Changing the roles, One-to-one conversations with a man or woman to speak about different topics e.g. sports, cinema, food, travel [7]. Milieu or the environment of patient's surrounding is impactful on the level of how he/she interacts with people within the environment. Young clients hospitalized for treatments are often dull as they feel that there is nothing left in their life and all colors have faded from their existence because of bad experiences of social life. Improves their perceptions the environmental changes are essential with planned changes. Favorite colors, posters or setting of furniture through keeping in mind the expert principles can help at very great extent [8]. Encouraging young clients into routine or daily physical tasks improves socializations. For example one is washing clothes and lacks washing powder needs to go into other person's room to borrow. Patients involved in cooking food, washing clothes, hygiene and sports might have positive chances of improving social interaction. Don't think if they are not following so we shouldn't encourage them [9]. Group therapies are also effective strategy for improving social interaction as they release the anxiety of an individual through looking towards and increased human interaction. Sitting with a group, listening to each other and participating with them enhance social inclusion among patients. The learn how to share their opinions [10]. In addition, flooding is an exposure therapy to stimuli that is often ignored or increases anxiety like in case of social inclusion planned, proper and sudden exposure to people with enhanced motivation can help young clients [11]. Providing them opportunities to do group works, or prepare speeches, talking to other residents can also boost young children's confidence [10].

\section{Conclusion}

Personally as care givers either at home or any health care setup, we should study deeply before applying these activities and collaborate with experts who can guide contextually relevant practices. Incubation spaces for the practical implication of these strategies should be provided and stimulating spots for young children who study, or work should also be developed. As young children are asset of our future and it is significant that social interaction should be promoted for their positive mental wellbeing and for learning common social practices of own context.

\section{References}

1. Boyd MA (2008) Psychiatric nursing: Contemporary practice. lippincott Williams \& wilkins.

2. Soomar SM (2016) Effective Methods of Coping with Academic Stress.

3. Videbeck S (2013) Psychiatric-mental health nursing. Lippincott Williams \& Wilkins.

4. Bor W, Dean AJ, Najman J, Hayatbakhsh R (2014) Are child and adolescent mental health problems increasing in the 21st century? A systematic review. Australian \& New Zealand Journal of Psychiatry 48(7): 606-616.

5. Fioravanti G, Dèttore D, Casale S (2012) Adolescent Internet addiction: testing the association between self-esteem, the perception of Internet attributes, and preference for online social interactions. Cyberpsychology, Behavior, and Social Networking 15(6): 318-323.

6. Ali TS, Krantz G, Gul R, Asad N, Johansson E, Mogren I (2011) Gender roles and their influence on life prospects for women in urban Karachi, Pak0istan: a qualitative study. Global health action 4(1): 7448.

7. Rus Calafell M, Gutiérrez Maldonado J, Ribas Sabaté J (2014) A virtual reality integrated program for improving social skills in patients with schizophrenia: A pilot study. Journal of behavior therapy and experimental psychiatry 45(1): 81-89.

8. Meghani MP (2016) Therapeutic milieu as an adjunct to pharmacological treatment in psychiatry.

9. Happell B, Platania Phung C, Scott D (2014) Proposed nurse-led initiatives in improving physical health of people with serious mental illness: A survey of nurses in mental health. Journal of clinical nursing 23(7-8): 1018-1029.

10. Webmed (2017) Group Therapy for Mental Health Problems.

11. Sundel M, Sundel SS (2017) Behavior change in the human services: Behavioral and cognitive principles and applications. Sage Publications.



